\title{
Off-Axis Electron Holography of Magnetic Nanowires and Chains, Rings, and Planar Arrays of Magnetic Nanoparticles
}

\author{
RAFAL E. DUNIN-BORKOWSKI, ${ }^{1,2 *}$ TAKESHI KASAMA,,${ }^{1,2}$ ALEXANDER WEI ${ }^{3}$ STEVEN L. TRIPP, ${ }^{3}$ \\ MARTIN J. HŸTCH, ${ }^{4}$ ETIENNE SNOECK, ${ }^{5}$ RICHARD J. HARRISON, ${ }^{6}$ AND ANDREW PUTNIS ${ }^{7}$ \\ ${ }^{1}$ Department of Materials Science and Metallurgy, University of Cambridge, Cambridge CB2 3QZ, United Kingdom \\ ${ }^{2}$ RIKEN (The Institute of Physical and Chemical Research), Advanced Research Laboratory, Hitachi Ltd., Hatoyama, \\ Saitama, 350-0395, Japan \\ ${ }^{3}$ Department of Chemistry, Purdue University, West Lafayette, Indiana 47907 \\ ${ }^{4}$ CECM-CNRS, 94407 Vitry-sur-Seine, France \\ ${ }^{5}$ CEMES-CNRS, 31055 Toulouse, France \\ ${ }^{6}$ Department of Earth Sciences, University of Cambridge, Downing Street, Cambridge CB2 3EQ, United Kingdom \\ ${ }^{7}$ Institut für Mineralogie, Universität Münster, D-48149 Münster, Germany
}

KEY WORDS magnetic induction mapping; electron holography; nanoparticles; nanowires

\begin{abstract}
A selection of recent results illustrating the application of off-axis electron holography to the study of magnetic microstructure in closely-spaced nanoparticles and nanowires is reviewed. Examples are taken from the characterization of FeNi nanoparticle chains, Co nanoparticle rings, two-dimensional arrays of naturally occurring magnetite crystals in minerals, and single crystalline Co nanowires. Approaches that can be used to separate the magnetic signal of interest from the mean inner potential contribution to the measured holographic phase shift are described, and the spatial and phase resolution that can be achieved are discussed. Microsc. Res.

Tech. 64:390-402, $2004 . \quad \odot 2004$ Wiley-Liss, Inc.
\end{abstract}

\section{INTRODUCTION}

The transmission electron microscope (TEM) is a powerful tool for characterizing the microstructure and chemistry of materials. However, most TEM imaging modes suffer from the drawback that information about the phase shift of the high-energy electron wave that has passed through the specimen is lost. The technique of electron holography, which is described in detail elsewhere (e.g., Dunin-Borkowski et al., 2004), overcomes this limitation by allowing the phase shift of the electron wave to be recovered. As the phase shift is sensitive to the in-plane component of the magnetic induction and the electrostatic potential in the specimen, an electron hologram can be used to provide quantitative information about magnetic and electric fields in materials at a spatial resolution that can approach the nanometer scale.

The TEM mode of off-axis electron holography is illustrated schematically in the form of a ray diagram in Figure 1. The specimen is examined using highly coherent illumination from a field emission gun (FEG) electron source with the region of interest positioned so that it covers approximately half the field of view. A (typically positive) voltage is applied to an electron biprism, which is located close to a conjugate image plane in the microscope, in order to tilt an electron wave that has passed through vacuum (or through a thin region of support film) with respect to a part of the same electron wave that has passed through the region of interest on the specimen. The two parts of the wave are then allowed to interfere to form a hologram in a slightly defocused image plane. When examining magnetic materials, holograms are normally recorded with the conventional microscope objective lens turned off, as its strong magnetic field would saturate the magne- tization in the specimen in the electron beam direction. A Lorentz lens (a high-strength mini-lens situated below the lower objective pole-piece) may then be used to record holograms at high magnification with the specimen in a field-free environment, with an optimal holographic overlap width and interference fringe spacing.

Neglecting dynamical diffraction (i.e., assuming that the specimen is thin and weakly diffracting), the measured phase shift can be expressed (in one dimension for simplicity) in the form

$$
\begin{aligned}
& \phi(x)=C_{E} \int V(x, z) d z-\left(\frac{e}{\hbar}\right) \int A_{z}(x, z) d z \\
&=C_{E} \int V(x, z) d z-\left(\frac{e}{\hbar}\right) \iint B_{\perp}(x, z) d x d z \\
& \text { where } C_{E}=\left(\frac{2 \pi}{\lambda}\right)\left(\frac{E+E_{0}}{E\left(E+2 E_{0}\right)}\right),
\end{aligned}
$$

$\mathrm{z}$ is the incident electron beam direction, $\mathrm{x}$ is a direction in the plane of the specimen, $A_{z}$ is the $\mathrm{z}$-component of

*Correspondence to: Rafal E. Dunin-Borkowski, Department of Materials Science and Metallurgy, University of Cambridge, Pembroke Street, Cambridge CB2 3QZ, UK. E-mail: rafal.db@msm.cam.ac.uk

Contract grant sponsor: Royal Society; Contract grant sponsor: EPSRC; Contract grant sponsor: National Science Foundation; Contract grant number 0243496-CHE; Contract grant sponsor: Department of Defense; Contract grant sponsor: Birck Nanotechnology Center at Purdue University; Contract grant sponsor: DFG; Contract grant sponsor: CNRS; Contract grant sponsor: European Community.

Received 20 January 2004; accepted in revised form 28 May 2004

DOI 10.1002/jemt.20098

Published online in Wiley InterScience (www.interscience.wiley.com). 


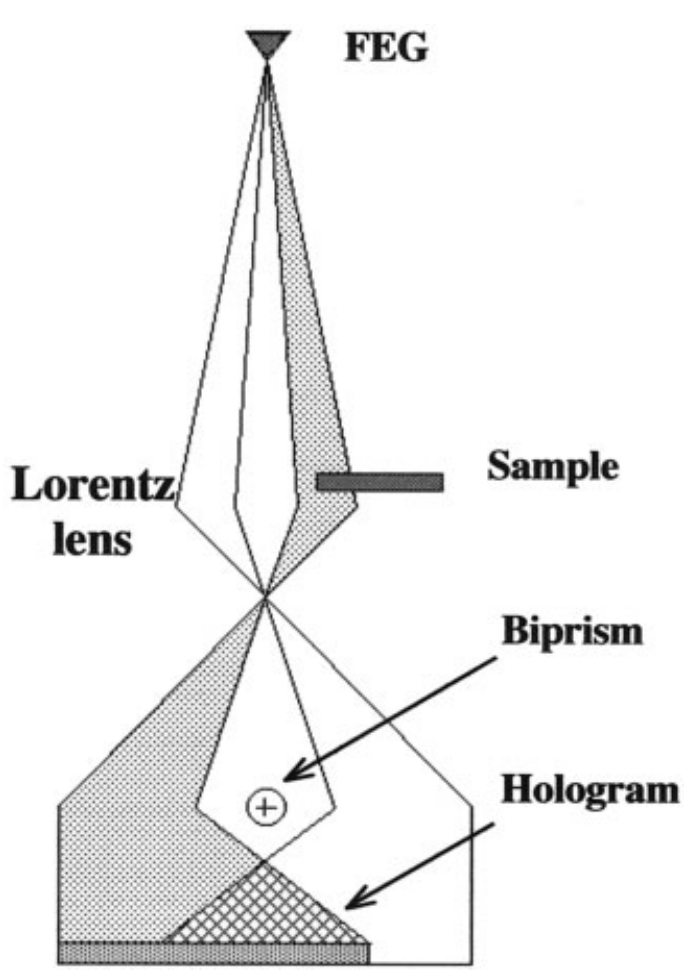

Fig. 1. Schematic illustration of setup used to generate off-axis electron holograms. The essential components are the field emission gun (FEG) electron source, which is used to provide coherent illumination, and the electron biprism, which is used to overlap the sample and reference waves. The Lorentz lens provides an optimal field of view and interference fringe spacing for electron holography of nanostructured magnetic materials, with the specimen imaged in a fieldfree environment.

the magnetic vector potential, $B_{\perp}$ is the component of the magnetic induction in the specimen perpendicular to both $\mathrm{x}$ and $\mathrm{z}, \mathrm{V}$ is the electrostatic potential, $\lambda$ is the electron wavelength, and $\mathrm{E}$ and $\mathrm{E}_{0}$ are, respectively, the kinetic and rest mass energies of the incident electron. The energy-dependent constant $\mathrm{C}_{\mathrm{E}}$ takes values of $7.29 \times 10^{6}$ and $6.53 \times 10^{6} \mathrm{rad} \mathrm{V}^{-1} \mathrm{~m}^{-1}$ at microscope accelerating voltages of 200 and $300 \mathrm{kV}$, respectively. It is straightforward to extend Equations 1 and 2 to two dimensions. In most magnetic materials, the potential $\mathrm{V}$ is associated solely with the mean inner potential (MIP) of the specimen $\mathrm{V}_{0}$, which depends on its composition and density. If neither $\mathrm{V}$ nor $\mathrm{B}_{\perp}$ vary in the beam direction, then Eq. (2) can be simplified to

$$
\phi(x)=C_{E} V(x) t(x)-\left(\frac{e}{\hbar}\right) \int B_{\perp}(x) t(x) d x
$$

where $t$ is the local specimen thickness. Differentiation with respect to $\mathrm{x}$ leads finally to

$$
\frac{\mathrm{d} \phi(\mathrm{x})}{\mathrm{dx}}=\mathrm{C}_{\mathrm{E}} \frac{\mathrm{d}}{\mathrm{dx}}\{\mathrm{V}(\mathrm{x}) \mathrm{t}(\mathrm{x})\}-\left(\frac{\mathrm{e}}{\hbar}\right) \mathrm{B}_{\perp}(\mathrm{x}) \mathrm{t}(\mathrm{x})
$$

In a specimen of uniform thickness and composition, the phase gradient

$$
\frac{\mathrm{d} \phi(\mathrm{x})}{\mathrm{dx}}=\left(\frac{\mathrm{et}}{\hbar}\right) \mathrm{B}_{\perp}(\mathrm{x})
$$

is directly proportional to the in-plane component of the magnetic induction, whose magnitude and direction can be depicted by adding contours to the phase image (or by displaying its cosine). Alternatively, straightforward algebra describing the differentiation of complex numbers can be used to show that the phase gradient can be evaluated directly from the real and imaginary parts of the reconstructed image wave $\operatorname{Re}(\psi)$ and $\operatorname{Im}(\psi)$ by using the relation

$$
\begin{aligned}
\frac{d \phi}{d x}=\left(\frac{\operatorname{Re}(\psi) \frac{d}{d x} \operatorname{Im}(\psi)-\operatorname{Im}(\psi) \frac{d}{d x}}{\operatorname{Re}(\psi)^{2}+\operatorname{Im}(\psi)^{2}}\right) & \\
& \equiv \operatorname{Im}\left(\frac{\frac{d}{d x} \psi}{\psi}\right) .
\end{aligned}
$$

When examining a magnetic nanostructure, the MIP contribution invariably dominates the measured phase shift. Although a holographic amplitude image could in principle be used to estimate the MIP contribution to the phase, the mean free path for inelastic scattering would need to be calibrated for each material in the specimen. In addition, an amplitude image is usually noisy and may contain strong diffraction or defocus contrast. A better approach involves recording a second hologram after inverting the specimen to change the sign of the magnetic contribution to the phase, and then using the sum and the difference of the two phase images to provide the magnetic and MIP contributions, respectively (Tonomura, 1992). Alternatively, two holograms of the specimen can be acquired at different microscope accelerating voltages. As the magnetic signal is independent of accelerating voltage, the difference between the two phase images can be used to provide the MIP contribution. However, in many practical situations it is neither feasible to turn the specimen over during an experiment nor convenient to change the accelerating voltage. An in situ magnetization reversal experiment may then be performed, with pairs of phase images subsequently selected that differ only in the (opposite) directions of magnetization in the specimen (Dunin-Borkowski et al., 1998). The magnetic and MIP contributions are again calculated by taking half the difference and half the sum of the phases of such pairs of holograms, respectively. More advanced approaches that can be used to eliminate specimen thickness as well as MIP effects have been developed for the examination of magnetic layers in cross-sectional geometry (Dunin-Borkowski et al., 1998)

It is important to note that a particular strength of electron holography is its ability to provide quantitative information about the magnetic properties of materials. For example, the magnetic moment of a nanoparticle can be obtained from the relation

$$
\mathrm{m}_{\mathrm{x}}=+\left(\frac{\hbar}{\mathrm{e}}\right) \int_{\mathrm{y}=-\infty}^{\mathrm{y}=+\infty} \int_{\mathrm{x}=-\infty}^{\mathrm{x}=+\infty} \frac{\partial}{\partial \mathrm{y}} \phi_{\operatorname{mag}}(\mathrm{x}, \mathrm{y}) \mathrm{dxdy},
$$


where $\phi_{\text {mag }}$ is the magnetic contribution to the phase shift and $\mathrm{y}$ is a direction perpendicular to $\mathrm{x}$ in the plane of the specimen (Dunin-Borkowski et al., 2001). According to Eq. 8, the magnetic moment in a given direction can be obtained simply by measuring the area under the first differential of $\phi_{\mathrm{mag}}$ evaluated in the perpendicular direction. The contribution of stray magnetic fields to the moment is included in this calculation if the integration is carried out over a large enough distance from the particle.

In this report, four of the most recent applications of electron holography to the characterization of magnetic fields in nanostructured materials are reviewed. The four examples are chosen to highlight the different approaches that can be used to separate a weak magnetic signal from a recorded phase image, as well as to illustrate the different magnetic properties of the materials. The work is motivated in part by the potential of using magnetic nanostructures with bistable domains and minimal crosstalk for information storage applications. The off-axis mode of electron holography is ideally suited to the characterization of such materials because unwanted contributions to the contrast from local variations in composition and specimen thickness can be removed from a phase image more easily than from images recorded using other phase contrast techniques in the TEM. For example, the Fresnel and Foucault modes of Lorentz microscopy and DPC (differential phase contrast) imaging provide signals that are approximately proportional to either the first or the second differential of the phase shift. These techniques inherently enhance contributions to the contrast from rapid variations in specimen thickness and composition, as compared to the weak and slowly varying magnetic signal. Earlier applications of electron holography to the characterization of magnetic structures and continuous films are not described. The reader is instead referred to previous studies describing the characterization of fine particles (Tonomura et al., 1980), recording media (Osakabe et al., 1983), the Aharonov-Bohm effect (Tonomura et al., 1986), superconducting vortices (Bonevich et al., 1993), hard magnets (McCartney and Zhu, 1998), patterned elements (Dunin-Borkowski et al., 2000), magnetotactic bacteria (Dunin-Borkowski et al., 2001), and charge order in manganites (Loudon et al., 2002).

\section{MATERIALS AND METHODS}

The results described below were acquired primarily using a Philips CM300ST FEG-TEM equipped with a rotatable electron biprism (a 0.6- $\mu$ m-diameter Aucoated quartz wire located in place of one of the conventional selected area apertures), a Lorentz mini-lens located in the bore of the objective lens pole-piece, and a 2,048-pixel CCD camera at the end of a Gatan imaging filter. A standard procedure was used to "reconstruct" each hologram, i.e., to obtain amplitude and phase information about the specimen wave. The intensity in an off-axis electron hologram (Fig. 2A and B) is the sum of the reference and image intensities and an additional set of cosinusoidal fringes whose phases and amplitudes are equal to the phase and amplitude of the specimen wave, respectively. The hologram is first Fourier transformed (Fig. 2C). The resulting complex image contains two "sidebands"; one is the Fourier

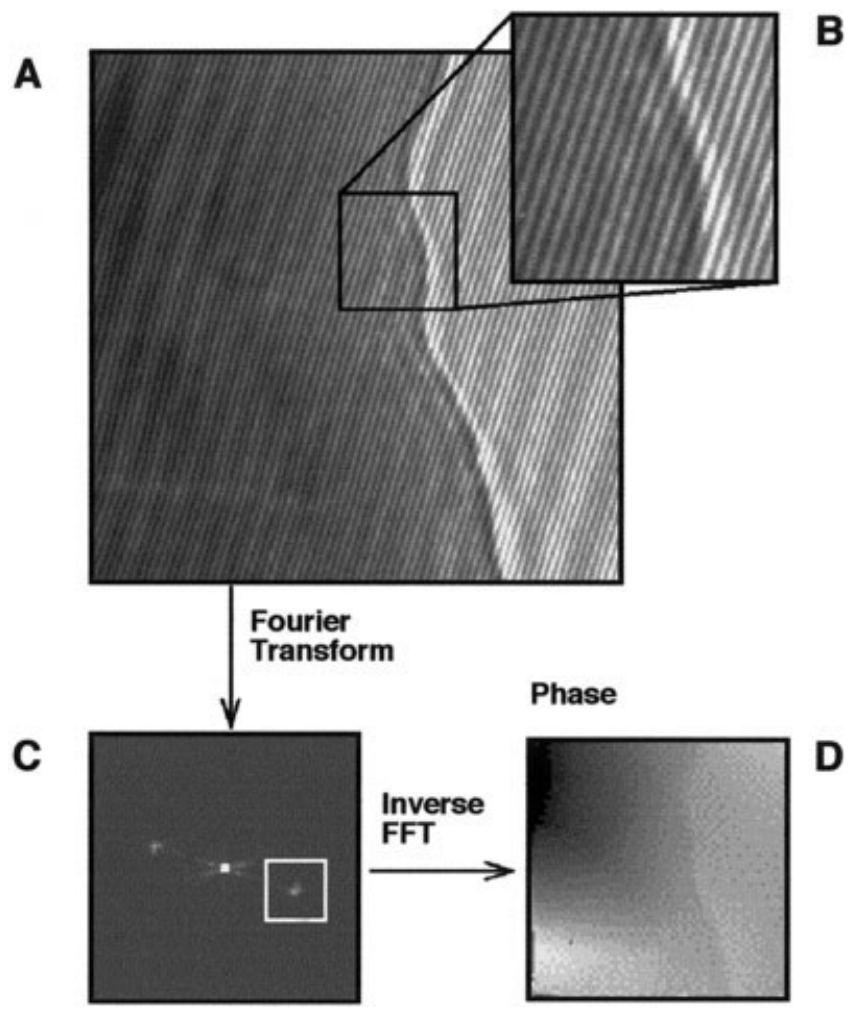

Fig. 2. A: Off-axis electron hologram from a thin crystal. B: Enlargement showing interference fringes within the specimen. C: Fourier transform of electron hologram. D: Phase image obtained after inverse Fourier transformation of the sideband marked with a box in $\mathrm{C}$.

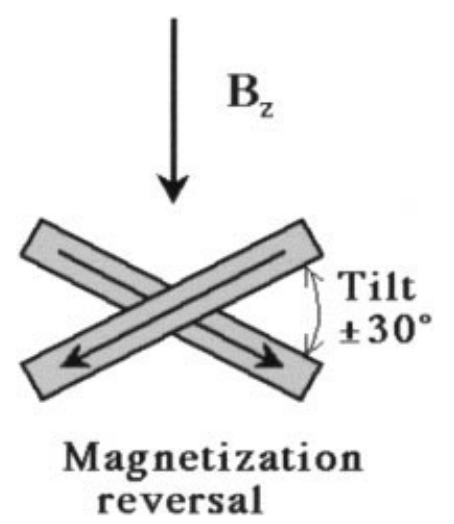

Fig. 3. Schematic diagram illustrating the use of specimen tilt to provide the in-plane component of the external field for in situ magnetization reversal experiments.

transform of the specimen wave, while the other is the Fourier transform of its complex conjugate. The reconstruction procedure involves selecting and inverse Fourier transforming one of these sidebands, as shown in Figure 2C and D. The phase and amplitude of the resulting complex image are then calculated. As the phase image is initially evaluated modulo $2 \pi$, phase discontinuities that are unrelated to specimen features must be removed using suitable algorithms (Ghiglia 

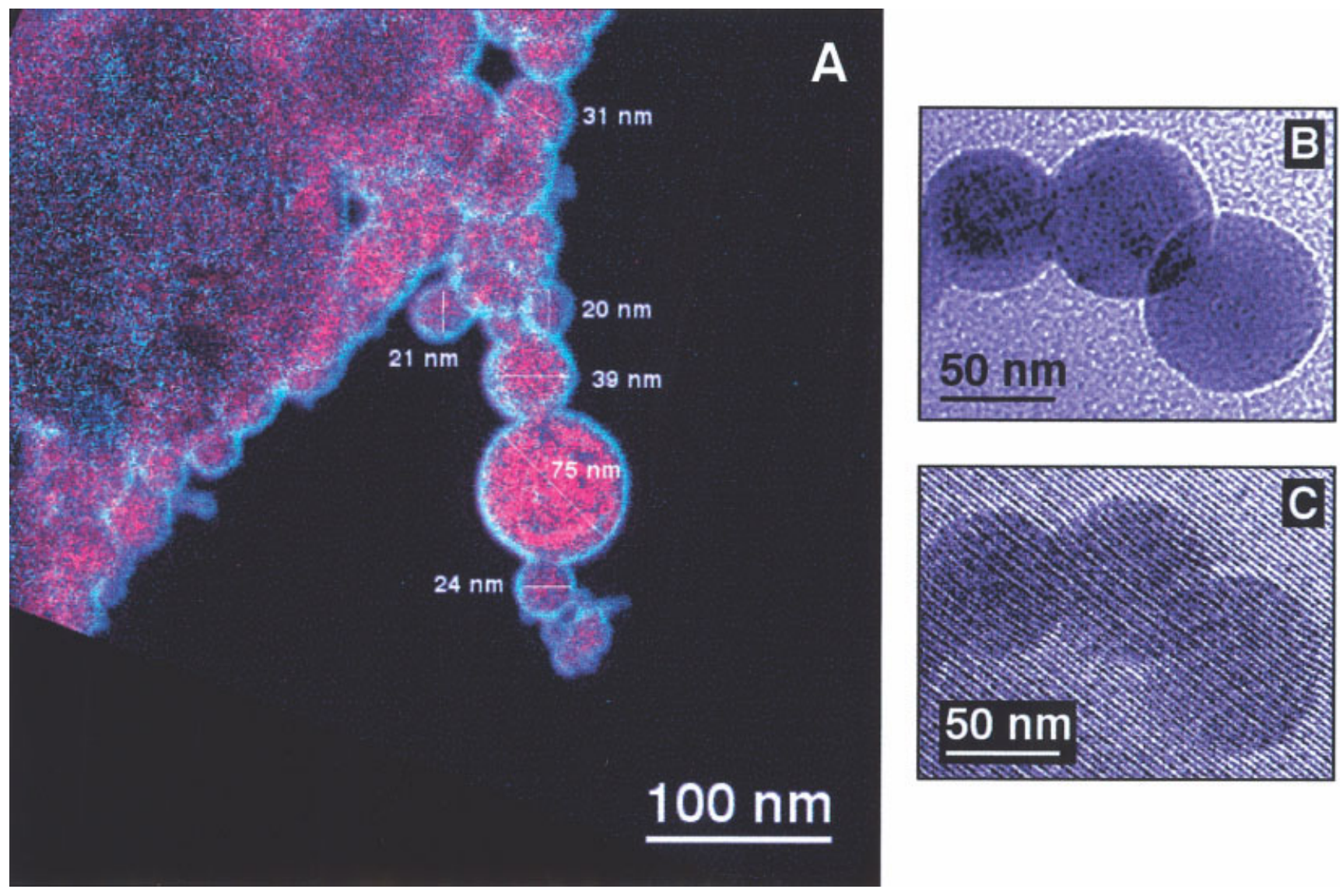

Fig. 4. A: Chemical map of $\mathrm{Fe}_{0.56} \mathrm{Ni}_{0.44}$ nanoparticles, obtained using three-window background-subtracted elemental mapping in a Gatan imaging filter, showing $\mathrm{Fe}$ (red), Ni (blue), and O (green). Bright-field image (B) and electron hologram $(\mathbf{C})$ of the end of a chain of $\mathrm{Fe}_{0.56} \mathrm{Ni}_{0.44}$ particles. Information about the magnetic induction in the sample is encoded in subtle changes in the positions of the holographic interference fringes. The hologram was recorded using an interference fringe spacing of $2.6 \mathrm{~nm}$. and Pritt, 1998) before reliable interpretation of image features becomes possible.

The Lorentz lens allows holograms to be recorded with the microscope objective lens switched off and the magnetic specimen located in a field-free environment. The objective lens can then be excited slightly and the specimen tilted (Fig. 3) to apply known magnetic fields to follow magnetization processes in situ in the TEM. Care is always required during analysis to assess the effect on the reference wave of long-range electromagnetic fields from the specimen (Matteucci et al., 1994). In addition, in the results presented below, reference holograms were always acquired from vacuum in order to take account of artifacts in the measured phase images (such as geometrical distortions due to the microscope projector lenses) associated with the imaging and recording system.

\section{RESULTS \\ FeNi Nanoparticle Chains}

The magnetic properties of nanoparticle chains have been studied for many years (e.g., Jacobs and Bean, 1955). However, there are few direct experimental measurements of the critical sizes at which such par- ticles are large enough to support magnetic vortices rather than single domains. Although electron holography has previously been applied to the characterization of magnetic nanoparticle chains (e.g., Seraphin et al., 1999; Signoretti et al., 2003), vortex states were never observed directly in these studies. Here, electron holography is used to characterize the magnetic microstructure of chains of ferromagnetic FeNi crystals, whose average diameter of $50 \mathrm{~nm}$ is expected to be close to the critical size for vortex formation (Hytch et al., 2003).

Figure $4 \mathrm{~A}$ shows a chemical map of a representative chain of $\mathrm{Fe}_{0.56} \mathrm{Ni}_{0.44}$ nanoparticles acquired using a Gatan imaging filter. The particles were prepared by condensing a vapor of the alloy rapidly in hexane (Champion and Bigot, 1996; Champion et al., 2003). They are compositionally homogeneous and are each coated in a 3-nm oxide shell. A defocused bright-field image and a corresponding electron hologram of part of a chain are shown in Figure $4 \mathrm{~B}$ and $\mathrm{C}$, respectively. The unwanted MIP contribution to the phase was determined by using the field of the microscope objective lens to magnetize each chain parallel and then antiparallel to its length in situ in the TEM, using the 
approach illustrated in Figure 3. In each case, the applied field was removed before recording phase images in field-free conditions. Each experiment was repeated several times to ensure that the inferred magnetization states were reproducible.

Figure 5A and $\mathrm{B}$ shows the remanent magnetic states of two chains of $\mathrm{Fe}_{0.56} \mathrm{Ni}_{0.44}$ particles, measured using electron holography. The contours, which have been generated from the magnetic contribution to the measured phase shift, provide a quantitative measure of the strength and direction of the local flux. Their density is proportional to the in-plane component of the induction in the specimen integrated in the electron beam direction. For a $75-\mathrm{nm} \mathrm{Fe}_{0.56} \mathrm{Ni}_{0.44}$ particle sandwiched between two smaller particles (Fig. 5A), closelyspaced contours run along the chain in a channel of width $22 \pm 4 \mathrm{~nm}$. A comparison of this result with micromagnetic simulations (Hytch et al., 2003) indicates that the particle contains a vortex whose axis is parallel to the chain axis, as shown schematically in Figure 5C. The lack of contours on either side of the particle results from the induction in these regions being substantially out-of-plane. The in-plane induction in these regions is also close to zero when integrated along the beam. In Figure 5B, a vortex can be seen end-on in a 71-nm particle at the end of a chain. The particle's neighbors appear to determine the handedness of the vortex, with the flux channel from the rest of the chain sweeping around the core to form concentric circles (Fig. 5D). The vortex core, which is now perpendicular to the chain axis, is only $9 \pm 2 \mathrm{~nm}$ in diameter. The larger value of $22 \mathrm{~nm}$ observed in Figure 5A results from dipole-dipole interactions along the chain. Vortices were never observed in particles below $30 \mathrm{~nm}$ in size, while intermediate states were observed in 30-70-nm particles.

In a phase image of a truly single domain spherical particle of radius a, a line profile through the center of the particle is predicted to take the form

$$
\begin{gathered}
\left.\phi(\mathrm{x})\right|_{\mathrm{x} \leq \mathrm{a}}=2 \mathrm{C}_{\mathrm{E}} \mathrm{V}_{0} \sqrt{\mathrm{a}^{2}-\mathrm{x}^{2}}+\left(\frac{\mathrm{e}}{\hbar}\right) \mathrm{B}_{\perp}\left[\frac{\mathrm{a}^{3}-\left(\mathrm{a}^{2}-\mathrm{x}^{2}\right)^{\frac{3}{2}}}{\mathrm{x}}\right] \\
\left.\phi(\mathrm{x})\right|_{\mathrm{x}>\mathrm{a}}=\left(\frac{\mathrm{e}}{\hbar}\right) \mathrm{B}_{\perp}\left(\frac{\mathrm{a}^{3}}{\mathrm{x}}\right)
\end{gathered}
$$

where $\mathrm{V}_{0}$ is the mean inner potential and $\mathrm{B}_{\perp}$ is the magnetic induction (de Graef et al., 1999). After some algebra, Eqs. 9 and 10 can be used to show that the height of the step in the magnetic contribution to the phase shift across a uniformly magnetized sphere is given by the expression

$$
\begin{aligned}
\Delta \phi_{\mathrm{MAG}} & =\left(\frac{2 \sqrt{2}\left(1-\left(1-\frac{\sqrt{3}}{2}\right)^{\frac{3}{2}}\right)}{3^{\frac{1}{4}}}\right)\left(\frac{\mathrm{e}}{\hbar}\right) \mathrm{B}_{\perp} \mathrm{a}^{2} \\
& \equiv 2.044\left(\frac{\mathrm{e}}{\hbar}\right) \mathrm{B}_{\perp} \mathrm{a}^{2},
\end{aligned}
$$
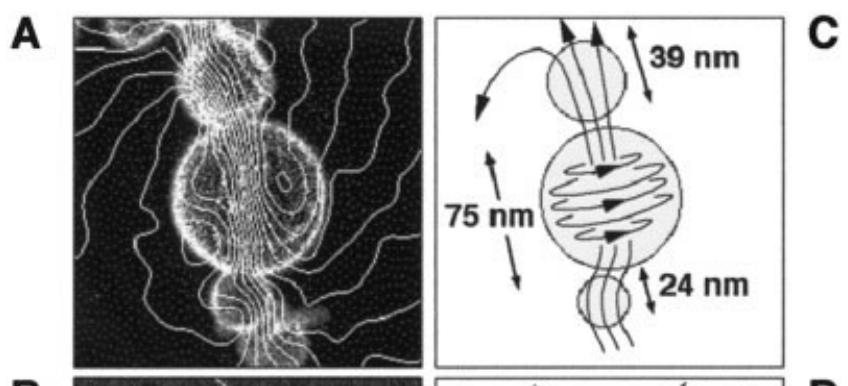

B
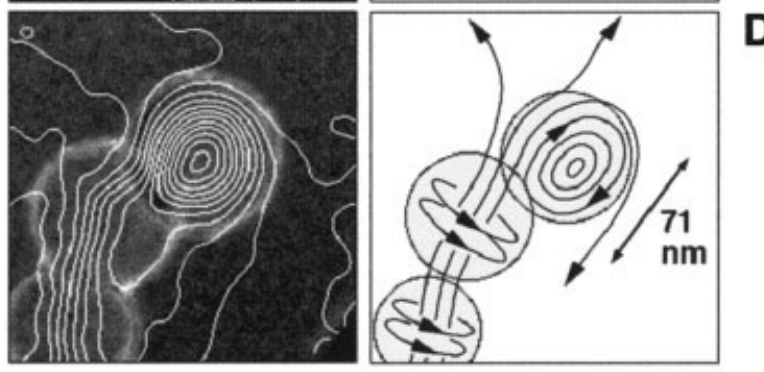

\section{$100 \mathrm{~nm}$}

Fig. 5. A,B: Experimental phase contours showing the strength of the local magnetic induction (integrated in the electron beam direction) in two different chains of $\mathrm{Fe}_{0.56} \mathrm{Ni}_{0.44}$ particles, recorded with the electron microscope objective lens switched off. The particle diameters are: (A) $75 \mathrm{~nm}$ between two smaller particles; (B) $71 \mathrm{~nm}$ at the end of a chain. Contours, whose spacings are 0.083 and 0.2 radians for images A and B, respectively, have been overlaid onto oxygen maps of the particles recorded using a Gatan imaging filter. The mean inner potential contribution to the phase has been removed from each image. C,D: Schematic representations of the magnetic microstructure in the chains. Magnetic vortices spinning about the chain axis are visible in $\mathrm{C}$ and $\mathrm{D}$. A vortex spinning perpendicular to the chain axis is also visible in D.

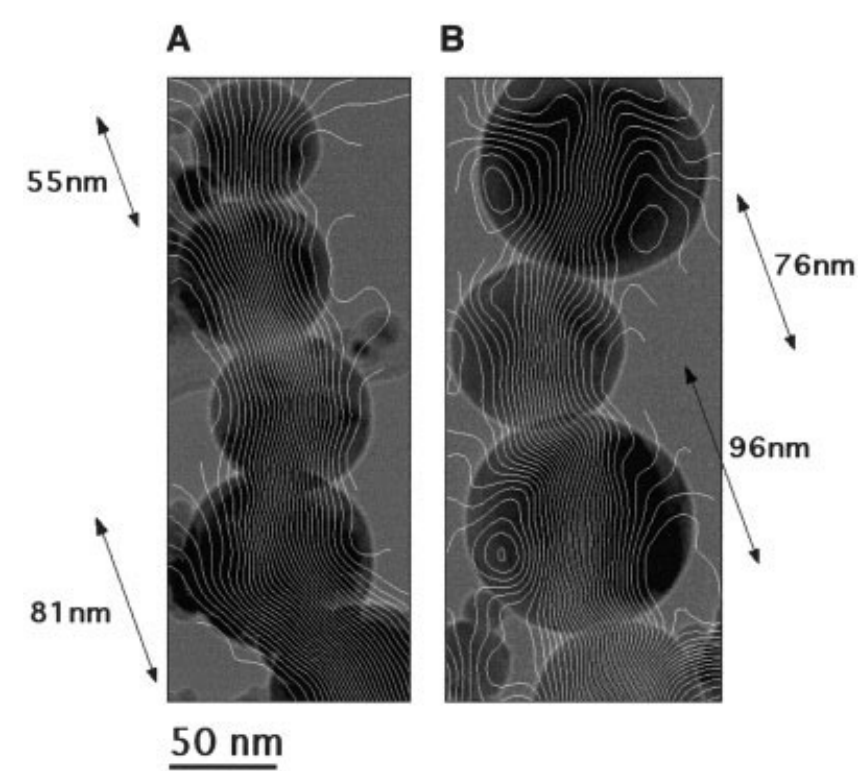

Fig. 6. A,B: Phase contours, overlaid onto bright-field images, showing the strength of the local magnetic induction in two chains of $\mathrm{Fe}_{010} \mathrm{Ni}_{090}$ particles, recorded with the electron microscope objective lens switched off. The contour spacing in each image, from which the mean inner potential contribution to the phase has been removed, is 0.125 radians. 


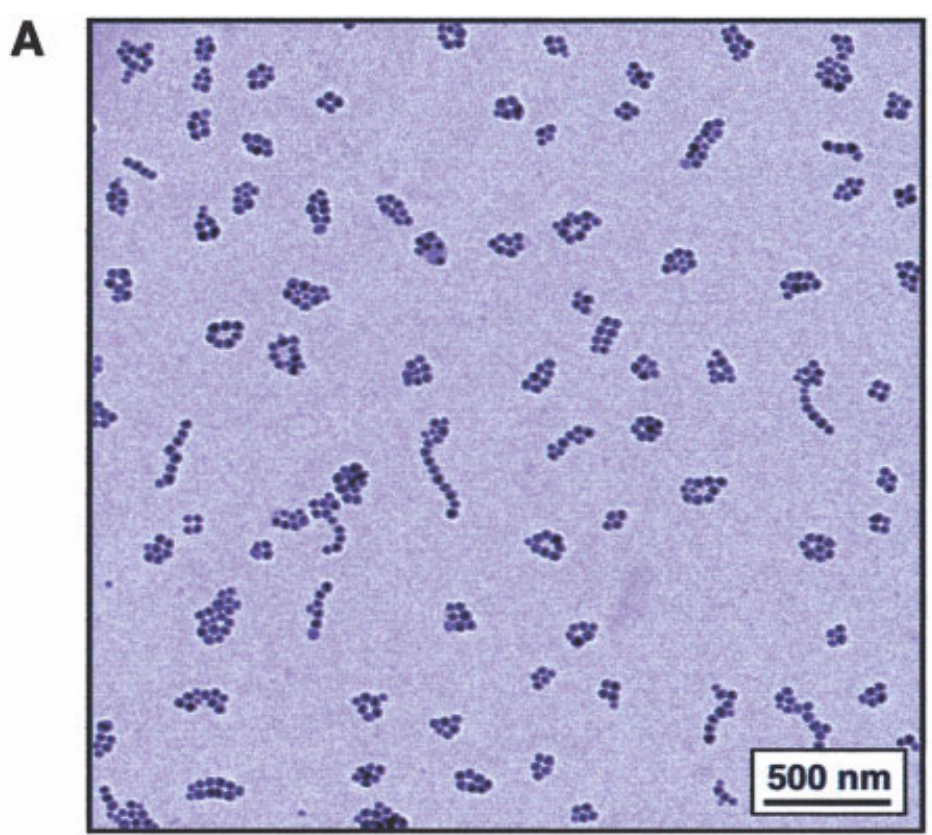

B
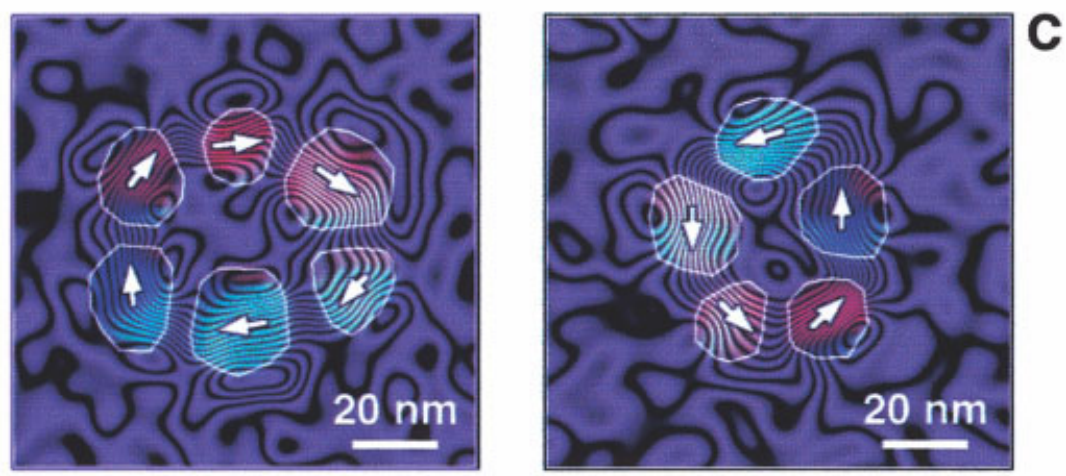

D
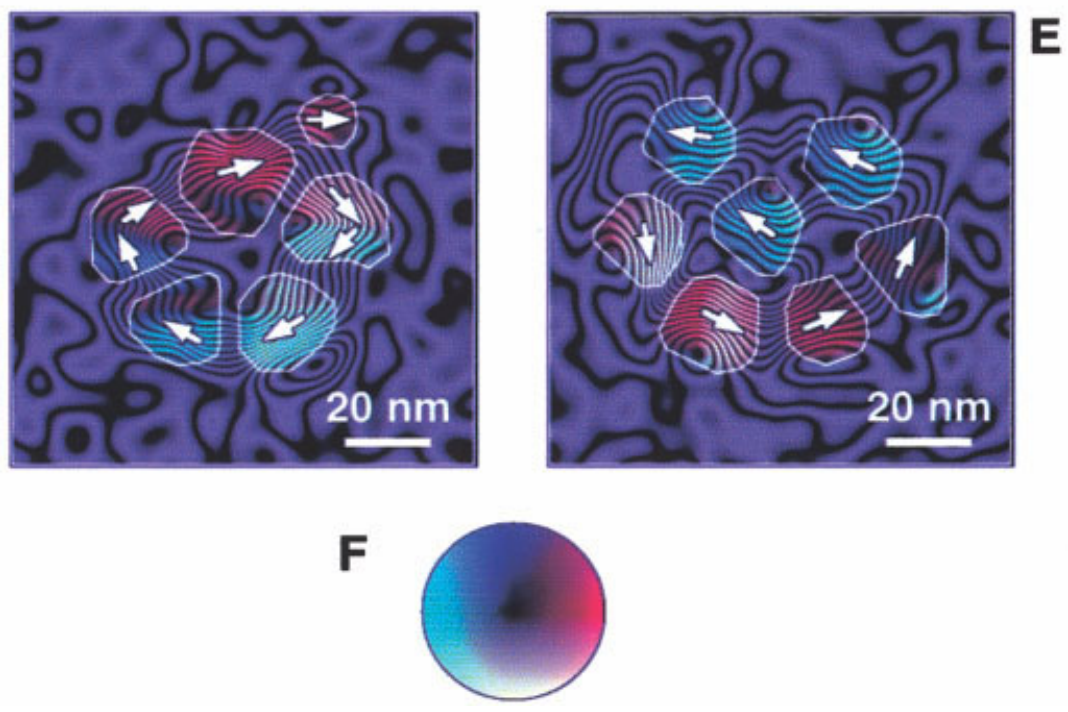

Fig. 7. A: Low-magnification bright-field image of self-assembled Co nanoparticle rings and chains deposited onto an amorphous carbon film. Each Co particle has a diameter of between 20 and $30 \mathrm{~nm}$. B-E: Magnetic phase contours $(128 \times$ amplification; 0.049 radian spacing), formed from the magnetic contribution to the measured phase shift, in four different nanoparticle rings. The outlines of the nanoparticles are marked in white, while the direction of the measured magnetic induction is indicated both using arrows and according to the color wheel shown in $\mathbf{F}($ red = right, yellow = down, green $=$ left, blue $=$ up). 
which can be contrasted with the equivalent expression for a uniformly magnetized cylinder of radius a, of

$$
\Delta \phi_{\mathrm{MAG}}=\pi\left(\frac{\mathrm{e}}{\hbar}\right) \mathrm{B}_{\perp} \mathrm{a}^{2} .
$$

These equations are strictly only valid for isolated particles in which the magnetization is uniform in magnitude and direction. Comparisons of these predictions with measurements of the step in the magnetic contribution to the phase across FeNi particles such as those in Figure 5 indicate that the measurements are consistent with Eq. 11 up to a particle size of $\sim 40 \mathrm{~nm}$. Above this size, the measured phase shifts are approximately equal because of the formation of flux channels, whose diameters are approximately independent of particle diameter.

The images shown in Figure 5 can be compared with similar induction maps obtained from chains of particles that have different alloy concentrations. Results from $\mathrm{Fe}_{0.10} \mathrm{Ni}_{0.90}$ particles are shown in Figure 6. The magnetic microstructure in these chains, which have a higher Ni concentration and a larger exchange length than $\mathrm{Fe}_{0.56} \mathrm{Ni}_{0.44}$, is closer to that expected for a chain of single-domain spheres. Flux channels in these particles have a diameter of $\sim 70 \mathrm{~nm}$ (e.g., the 96-nmdiameter particle in Fig. 6B), which is larger than the diameter of $22 \mathrm{~nm}$ observed for $\mathrm{Fe}_{0.56} \mathrm{Ni}_{0.44}$. The flux channels now form only when the particles are above $\sim 100 \mathrm{~nm}$ in size.

The complicated domain structures observed in Figures 5 and 6 result in part from the presence of magnetostatic interactions between each particle and its neighbors, which stabilize the vortices. Although predictions based on the magnetostatic energy of a uniformly magnetized sphere (Bertotti, 1998) and on spins curling around an easy axis with a null axial component (O'Handley, 2000) can be used to calculate critical particle diameters for the formation of flux channels, these predictions are not strictly valid for nanoparticle chains. The diameters of the vortex cores depend sensitively on their orientation with respect to the chain axis. The complexity of the vortex states highlights the importance of controlling the shapes, sizes, and positions of closely-spaced magnetic nanocrystals for applications such as non-volatile storage devices.

\section{Co Nanoparticle Rings}

An illustration of magnetostatic interactions between particles that are each single domain is provided by the examination of rings (bracelets) of 20-nm-diameter Co particles. Such rings are appealing candidates for high-density information storage applications because they are expected to form chiral domain states that exhibit flux closure (FC). Such FC states were
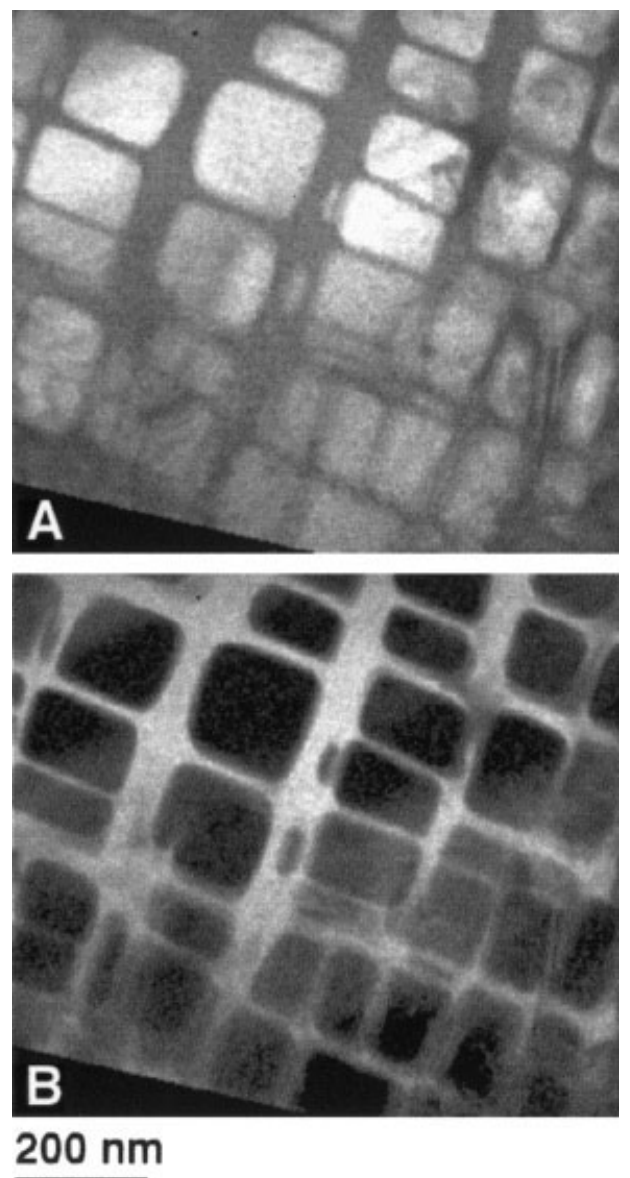

Fig. 8. Three-window background-subtracted elemental maps acquired from a naturally occurring titanomagnetite sample with a Gatan imaging filter using (A) the Fe L edge, and (B) the Ti L edge. Brighter contrast indicates a higher concentration of $\mathrm{Fe}$ and $\mathrm{Ti}$ in $\mathrm{A}$ and $\mathrm{B}$, respectively.

used in early data storage applications involving ferrite cores, and have recently been proposed as elements in novel device architectures for magnetoresistive random-access memory (e.g., Zhu et al., 2000). Nanoparticle rings are also of interest for the development of electron holography because their magnetization directions cannot be reversed through the application of in-plane fields. As a result, in the present study phase images were obtained both before and after turning the specimen over. The resulting pairs of phase images were aligned in position and angle, and their sum and difference calculated as described above.

Figure 7A shows a low-magnification bright-field image of the Co rings, which have dimensions that are
Fig. 9. Magnetic phase contours from the region shown in Figure 8, measured using electron holography. Each image was acquired with the specimen in field free conditions. The outlines of the magnetiterich regions are marked in white, while the direction of the measured magnetic induction is indicated both using arrows and according to the color wheel shown at the bottom of the figure (red = right, yellow $=$ down, green $=$ left, blue $=$ up). A,C,E,G: Obtained after applying a large $(>10,000$ Oe) field towards the top left, then the indicated field towards the bottom right, after which the external magnetic field was removed for hologram acquisition. B,D,F,H: Obtained after applying identical fields in the opposite directions. 

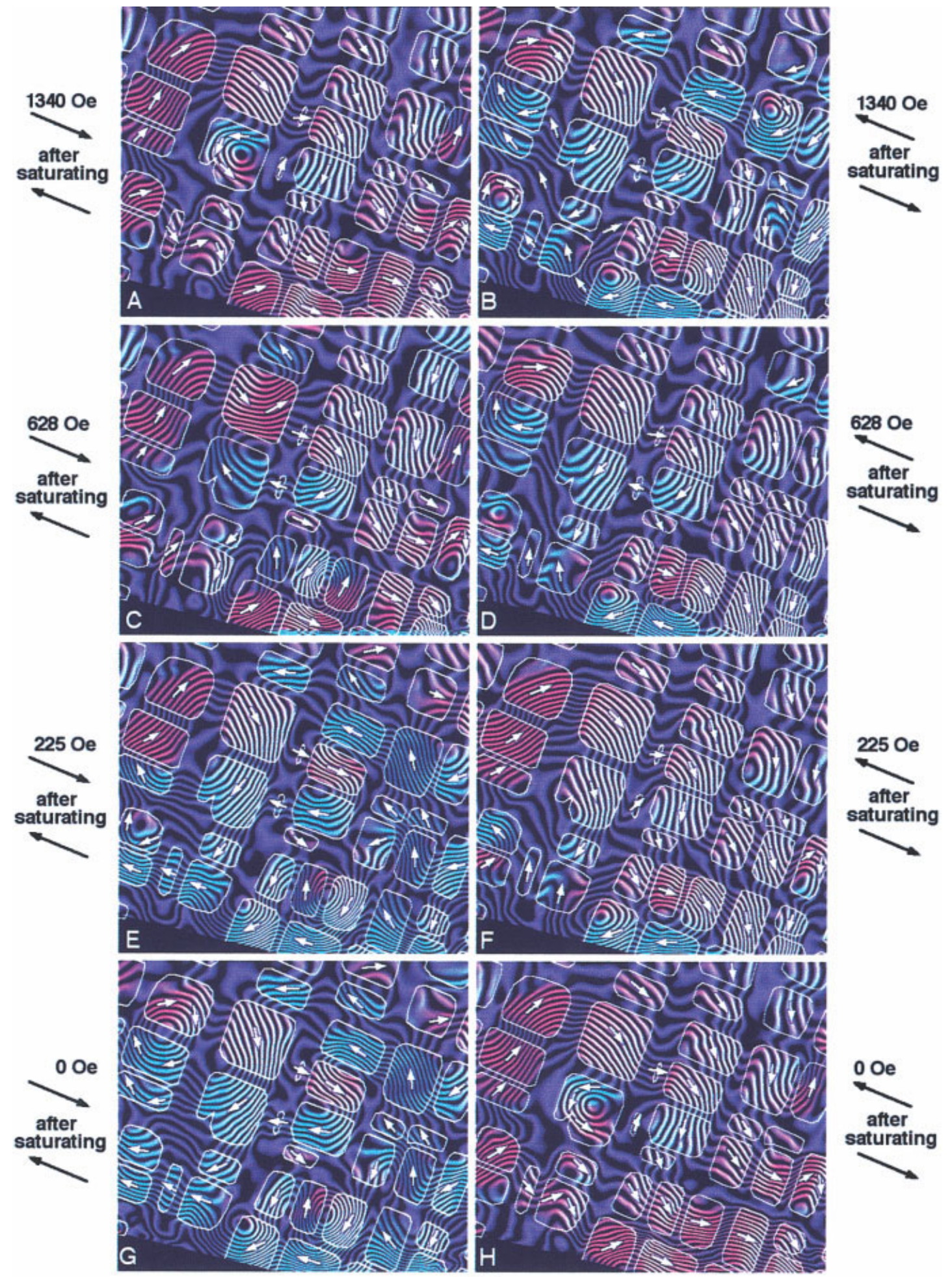
$200 \mathrm{~nm}$
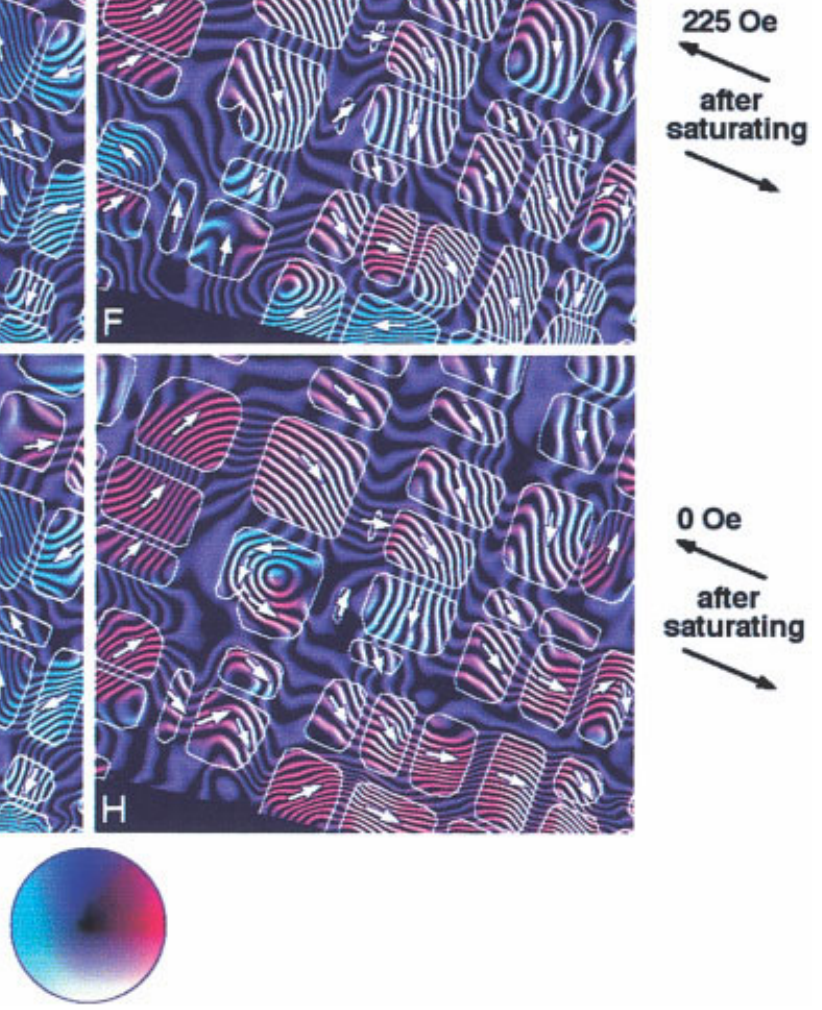

Fig. 9. 
well below the limits of conventional lithography (Tripp et al., 2002). A variety of self-assembled structures is visible, including five- and six-particle rings, chains and close-packed aggregates containing three or more particles. The particles are each encapsulated in a 3-4-nm oxide shell. Figure 7B-D shows magnetic FC states in four different Co particle rings, recorded using electron holography at room temperature in zero-field conditions (Tripp et al., 2003). The magnetic flux lines, which are formed from the cosine of 128 times the magnetic contribution to the measured phase shift, reveal the in-plane induction within each ring ensemble. Magnetic force microscopy confirmed the absence of magnetic vortex cores, such as those observed in planar magnetic disks (e.g., Wachowiak et al., 2002).

Our results showed that the specimen contained an approximately 50:50 mixture of clockwise and counterclockwise ground-state configurations, to which the rings relaxed after exposure to a 2 T out-of-plane magnetic field. The stability and reproducibility of the states suggests that even smaller particles may form stable FC states at room temperature, in contrast to vortex states in magnetic disks, which are only stable for diameters of above $\sim 100 \mathrm{~nm}$ (Cowburn et al., 1999). Most recently, we have used electron holography to demonstrate that the chirality of the FC states can be switched using an out-of-plane magnetic field. Although the critical field strength for magnetization reversal remains to be determined, preliminary observations show that FC states in 4- to 7-membered rings can be reversed reproducibly using an out-of-plane field of 1,600 Oe. The reversal mechanism is highly complex, and will be discussed elsewhere.

\section{Planar Arrays of Magnetite Nanoparticles}

The magnetic behavior of chains and rings of nanomagnets described above contrasts with that of a regular two-dimensional array of closely-spaced crystals. A striking example of such an array is found in nature, in the titanomagnetite mineral system (Dunlop and Özdemir, 1997). Solid-state processes such as subsolvus exsolution, which can generate single domain (SD) magnetite particles from multi-domain (MD) grains, are thought to be responsible for the strong and stable remanent magnetization exhibited by many rocks, and have been proposed as a possible source of magnetic anomalies on Mars (Acuña et al., 1999; Connerney et al., 1999). Figure $8 \mathrm{~A}$ and $\mathrm{B}$ shows chemical maps of a crystalline region of a finely-exsolved magnetiteulvöspinel $\left(\mathrm{Fe}_{3} \mathrm{O}_{4}-\mathrm{Fe}_{2} \mathrm{TiO}_{4}\right)$ specimen, in which intermediate compositions (termed titanomagnetites) exsolve during slow cooling to yield an intergrowth of magnetite-rich blocks separated by non-magnetic ulvöspinel-rich lamellae (Price, 1981). The Fe and Ti L maps shown in Figure 8 were obtained using threewindow background-subtracted elemental mapping in a Gatan imaging filter. Ulvöspinel-rich exsolution lamellae subdivide the titanomagnetite grain into a fairly regular array of magnetite-rich blocks. The specimen thickness increases from $70 \mathrm{~nm}$ at the top of the region shown to $195 \mathrm{~nm}$ at the bottom. The magnetite blocks are, therefore, roughly equidimensional.

Remanent states were recorded by tilting the specimen to the maximum available angle of $41^{\circ}$ in zero field and turning the objective lens on fully to saturate the sample. This provided a known starting point from which further fields could be applied. The objective lens was then turned off, the specimen tilted to $41^{\circ}$ in zero field in the opposite direction and the objective lens excited partially to apply a known in-plane component of magnetic field to the specimen in the opposite direction. The objective lens was switched off and the sample tilted back to $0^{\circ}$ in zero field to record the hologram. This procedure was repeated for a number of different applied fields (Harrison et al., 2002).

For this specimen, mean inner potential contributions to the measured phase shifts had to be removed using a different procedure to that used for the $\mathrm{FeNi}$ and Co nanoparticles described above. Although both thickness and composition vary in the magnetite-ulvöspinel specimen, the different compositions of magnetite and ulvöspinel are compensated by their densities in such a way that their MIPs are exactly equal. Hence, only a thickness correction is required. The local specimen thickness $t$ across the region of interest was determined from $t / \lambda_{i}$ maps obtained using energyfiltered imaging (where $\lambda_{i}$ is the inelastic mean-freepath, and is determined experimentally to be $170 \mathrm{~nm}$ for the present sample at $300 \mathrm{kV}$ from a comparison of energy-filtered and phase images). The constant of proportionality between $t / \lambda_{i}$ and the MIP contribution to the phase was determined by least-squares fitting to data collected near the edge of the wedge-shaped sample, where the magnetic contribution is negligible. Since the thickness of the sample varied smoothly and slowly over the region of interest, this procedure was found to provide a very good method of separating the magnetic contribution from the measured phase images.

Figure 9 shows eight remanent magnetic states recorded using electron holography after applying the in-plane fields indicated in the figure. (For in-plane fields of $1,340,628$, and 225 Oe, with the sample tilted by $41^{\circ}$, the out-of-plane component of the applied field is 1,520, 713, and 255 Oe, respectively). As in Figure 7 above, the black contour lines provide the direction and magnitude of the magnetic induction in the plane of the sample, which can be correlated with the positions of the magnetite blocks (outlined in white). The direction of the induction is also indicated using colors and arrows, according to the color wheel shown at the bottom of the figure. Figure 8 shows that the magnetic domain structure in this sample is extremely complex. Although the magnetization is never saturated in the direction of the applied field, analysis shows that the average magnetization direction is parallel to this direction. The contours outside the blocks are associated with stray interaction fields and are, on average, more widely spaced than the internal contours.

In Figure 9, the smallest block observed to form a vortex is larger than the predicted minimum size of $70 \mathrm{~nm}$ for vortices to form in isolated cubes of magnetite. The abundance of SD states implies that they have a lower energy than vortex states in the presence of strong interactions. For isolated cubes of magnetite, micromagnetic simulations (Williams and Wright, 1998) predict the opposite, with the vortex state having a lower energy than the SD state over the size range 70-200 $\mathrm{nm}$. The demagnetizing energy, which nor- 
A

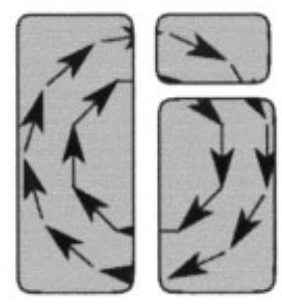

B

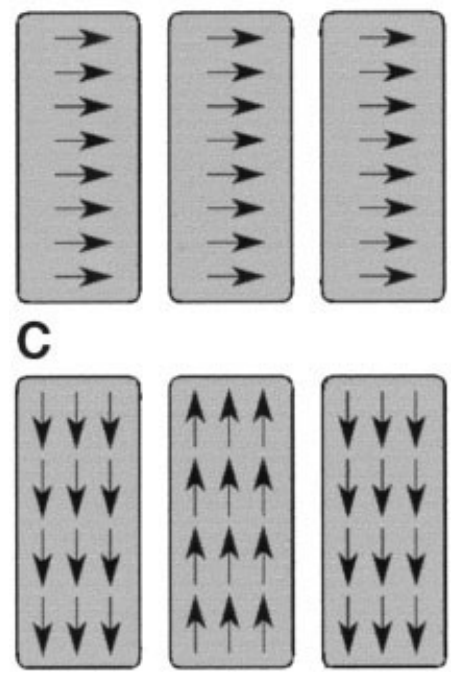

Fig. 10. A-C: Schematic diagrams showing some of the possible magnetization states of three closely-spaced regions of magnetic material in the titanomagnetite specimen shown in Figure 9.

mally destabilizes the SD state with respect to the vortex state in isolated particles, is greatly reduced in an array of strongly interacting particles. Great care must clearly be taken if simulations of isolated particles are used to predict the domain states of interacting particles. In the present study, the magnetization directions of the blocks are determined primarily by shape anisotropy and interactions. The influence of magnetocrystalline anisotropy is relatively small.

In Figure 9, several blocks are observed to act collectively to form magnetic "superstates" that would normally be observed in larger magnetized regions. A common example is where two or more blocks interact to form a vortex, as shown schematically in Figure 10A. A second example of collective behavior involves the interaction of a chain of blocks to form an SD superstate magnetized parallel to the chain axis but perpendicular to the easy axes of the blocks. This is illustrated schematically in Figure $10 \mathrm{~B}$ and can be found in several places in Figure 9 (e.g., at the bottom of Fig. 9A, B, $\mathrm{D}$, and $\mathrm{H}$ ). If the three blocks are instead magnetized perpendicular to the chain axis, then a third superstate equivalent to a three-domain particle is generated (Fig. 10C). The central block is now magnetized antiparallel to the blocks on either side of it. Several examples of this behavior can be found (e.g., at the bottom of Fig. 9C, E, and G).
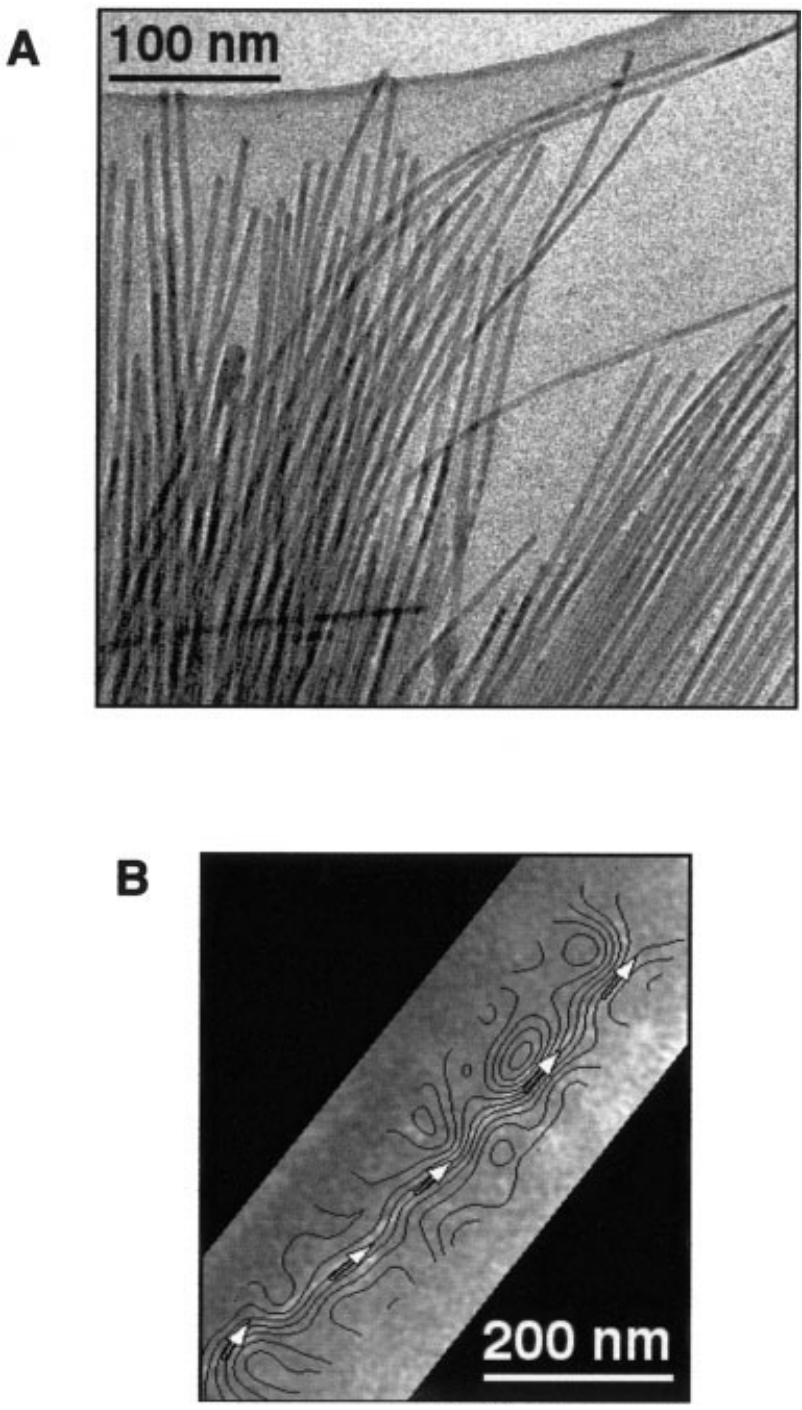

Fig. 11. A: Bright-field image of the end of a bundle of Co nanowires adjacent to a hole in the carbon support film. B: Contours (0.005 radian spacing) generated from the magnetic contribution to the phase shift of a single isolated Co nanowire, superimposed onto the mean inner potential contribution to the measured phase shift.

\section{Co Nanowires}

An important question relates to the minimum size of a nanostructure in which magnetic fields can be characterized successfully using electron holography. This point is now addressed through the characterization of 4-nm-diameter single crystalline Co nanowires (Snoeck et al., 2003). The difficulty of the problem results from the fact that, according to Eqs. 4 and 12, the MIP contribution to the phase shift at the center of a $4-n m$ wire relative to that in vacuum is 0.57 radians (assuming a value for $\mathrm{V}_{0}$ of $22 \mathrm{~V}$ ), whereas the step in the magnetic contribution to the phase shift across the wire is only 0.032 radians (assuming a value for $B$ of $1.6 \mathrm{~T})$. Electron holography has previously only been used to characterize magnetic wires of significantly larger diameter (Beeli et al., 1997; Sugawara et al., 
1998), for which the separation of the magnetic signal from the total phase shift was not required.

Figure 11A shows a TEM bright-field image of a bundle of 4-nm-diameter Co wires, which were prepared by organometallic decomposition and are each between a few hundred $\mathrm{nm}$ and several hundred $\mu \mathrm{m}$ in length. As for the FeNi nanoparticle chains described above, the magnetic contribution to the phase shift was obtained by recording two holograms of each area of interest, between which the wires were magnetized parallel and then antiparallel to their length by tilting the sample by $\pm 30^{\circ}$ about an axis perpendicular to the wire axis and using the conventional microscope objective lens to apply a large in-plane field to the specimen. The lens was then switched off and the sample returned to zero tilt to record each electron hologram. This procedure relies on the ability to reverse the magnetization in the sample exactly, which is a good assumption for the highly anisotropic and narrow wires.

Figure 11B shows the magnetic contribution to the measured phase shift for an isolated wire in the form of contours, which are spaced 0.005 radians apart. The contours have been overlapped onto an image showing the MIP contribution to the phase shift so that they can be correlated with the position of the wire. The magnetic signal is very weak and noisy, and was smoothed before forming the contours; the resulting spatial resolution is estimated to be several tens of $\mathrm{nm}$. (The features in this image appear larger than their true dimensions because of the undersampling inherent in the holographic reconstruction procedure, as well as the need to smooth the measured phases digitally to reduce the effects of noise). The closely-spaced contours along the length of the wire confirm that it is magnetized along its axis. Although the magnetocrystalline easy axis coincides with the wire axis, the fact that the wire is magnetized along its length results primarily from its diameter and aspect ratio. The fact that the contours are not straight in Figure $11 \mathrm{~B}$ is intriguing. However, this effect may result from smoothing a signal that is noisy and weak.

Figure 12A shows a montage of three holograms obtained close to the end of a bundle of Co wires, which was magnetized approximately parallel to its length. The magnetic contribution to the measured phase shift is shown in Figure 12B in the form of contours, which are spaced 0.25 radians apart. As for the isolated wire in Figure 11B, the contours have been overlapped onto an image of the MIP contribution to the phase shift. The wires channel the magnetic flux efficiently along their length, and they fan out as the field decreases in strength at the end of the bundle. Although the signal from the bundle appears to obscure that from individual wires and junctions, these details can be recovered by increasing the density of the contours (Snoeck et al., 2003). The slight asymmetry between the contours on either side of the bundle in Figure 12B may result from the fact that the reference wave is affected by the magnetic leakage field of the bundle, which acts collectively as though it were a single wire of larger diameter. The step in phase across the bundle is $(9.0 \pm 0.2)$ radians, which is consistent with the presence of $(280 \pm 7)$ ferromagnetically coupled wires.
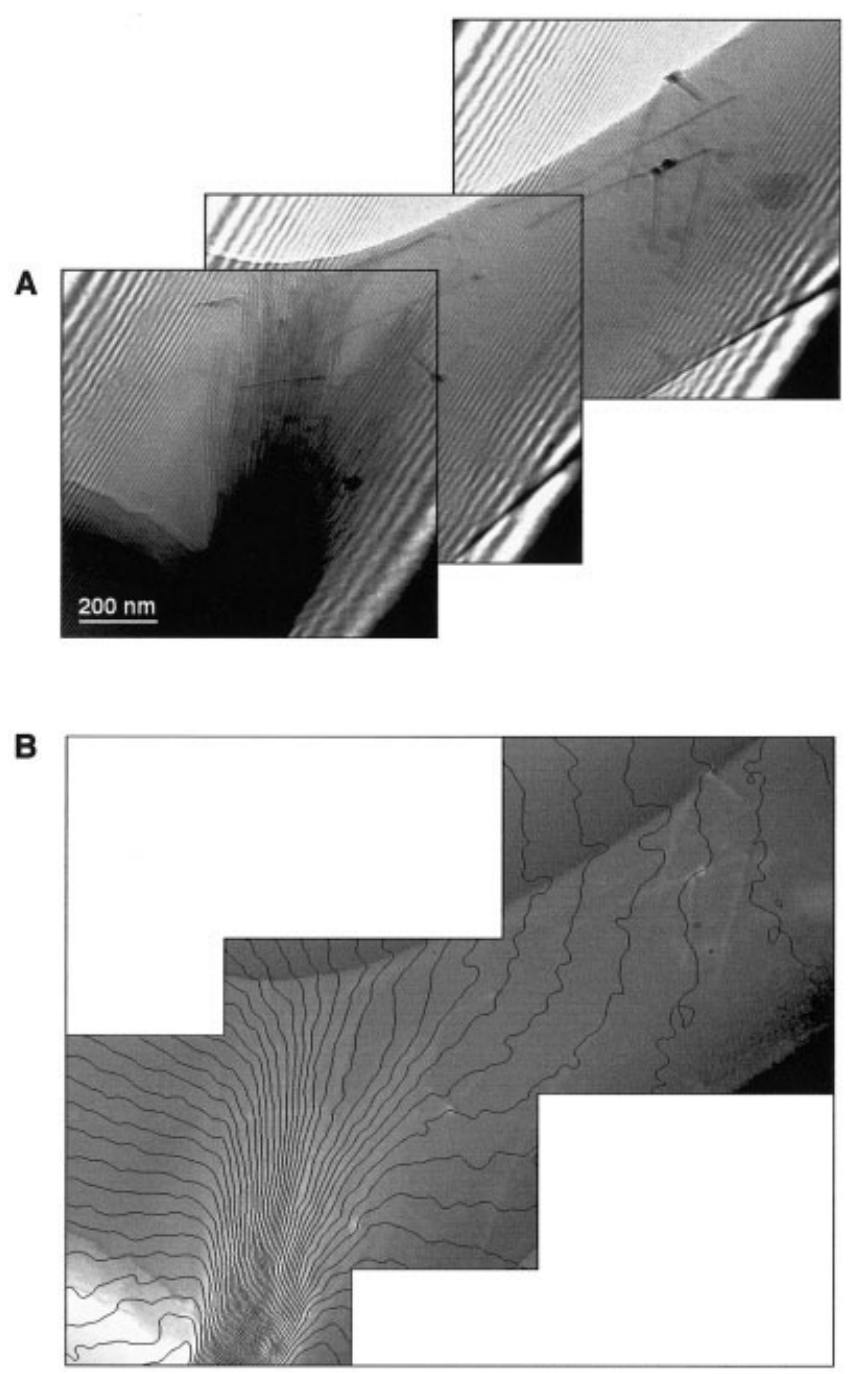

Fig. 12. A: Montage of three holograms of the end of a bundle of Co nanowires. The biprism voltage is $210 \mathrm{~V}$, the acquisition time for each hologram $16 \mathrm{~s}$, the holographic interference fringe spacing $3.9 \mathrm{~nm}$, and the holographic overlap width $1,160 \mathrm{~nm}$. No objective aperture was used. B: Magnetic remanent state, displayed in the form of contours $(0.25$ radian spacing) that have been generated from the measured magnetic contribution to the electron holographic phase shift, after saturating the wires in the direction of the bundle. The contours are superimposed onto the mean inner potential contribution to the phase shift.

\section{DISCUSSION AND CONCLUSIONS}

As magnetic nanostructures decrease in size, so electron holography provides an increasingly unique approach for characterizing their magnetic properties at high spatial resolution. The spatial resolution that can be achieved in a magnetic induction map is determined primarily by the spacing of the holographic interference fringes. However, the contrast of these fringes decreases as their spacing is reduced, and the recording process is also dominated by Poisson-distributed shot noise (Lichte et al., 1987). These parameters are in turn affected by the illumination diameter, exposure time, and biprism voltage. The minimum phase differ- 
ence between two pixels that can be detected is given by the expression

$$
\Delta \phi_{\min }=\frac{\mathrm{SNR}}{\mu} \sqrt{\frac{2}{\mathrm{~N}_{\mathrm{el}}}},
$$

where SNR is the signal-to-noise ratio in the phase image, $\mu$ is the holographic fringe visibility, and $\mathrm{N}_{\mathrm{el}}$ is the number of electrons per pixel (Lichte, 1995). In practice, averaging of the measured phase image is often used, particularly if the features of interest vary slowly across the image or in one direction. The final "phase resolution" (Harscher and Lichte, 1996) and "spatial resolution" are always inherently linked, in the sense that a small phase shift can be measured to high precision with poor spatial resolution, or with poor precision but high spatial resolution. In each of the examples described in this report, the recorded phase images were always smoothed slightly to remove noise, and the spatial resolution of the magnetic information was typically estimated to be between 10 and $20 \mathrm{~nm}$. This procedure is necessarily subjective, and great care is required to ensure that artifacts are not introduced. In future studies, higher spatial and phase resolution may be achieved by recording several holograms of each area of interest and subsequently averaging the calculated phase images.

Future developments in electron holography of nanostructured magnetic materials are likely to include approaches for combining electron holography with electron tomography (Midgley et al., 2001) to record magnetic vector fields in three dimensions inside materials, as well as the stray fields outside them (Lai et al., 1994). The application of tomography to the characterization of magnetic vector fields requires two high-tilt series of holograms to be recorded about orthogonal axes to reconstruct the three-dimensional distribution of two of the three components of the magnetic field in the sample. The third component can then be evaluated by making use of the criterion that div.B must equal zero everywhere. The primary challenge is then to subtract the MIP contribution from the phase shift measured at every tilt angle. Ultimately, with a sufficient investment of resources into this technique, both the acquisition and the reconstruction of such datasets could in principle be automated.

\section{ACKNOWLEDGMENTS}

We are grateful to Prof. M.R. Scheinfein and Dr. J. Loudon for discussions. R.E.D.B. thanks the Royal Society and the EPSRC for financial support. A.W. and S.L.T. gratefully acknowledge support from the National Science Foundation (0243496-CHE), the Department of Defense, and the Birck Nanotechnology Center at Purdue University. R.H. and A.P. thank the DFG for support. M.J.H., E.S., and R.E.D.B. are grateful to the CNRS and the European Community for support through a European research network (GDR-E) entitled "Quantification and Measurement in Transmission Electron Microscopy," grouping laboratories in France, the United Kingdom, Germany, and Switzerland.

\section{REFERENCES}

Acuña MH, Connerney JEP, Ness NF, Lin RP, Mitchell D, Carlson CW, McFadden J, Anderson KA, Reme H, Mazelle C, Vignes D,
Wasilewski P, Cloutier P. 1999. Global distribution of crustal magnetization discovered by the Mars Global Surveyor MAG/ER experiment. Science 284:790-793.

Beeli C, Doudin B, Ansermet JP, Stadelmann PA. 1997. Measurement of the remanent magnetization of single $\mathrm{Co} / \mathrm{Cu}$ and Ni nanowires by off-axis TEM electron holography. Ultramicroscopy 67:143-151.

Bertotti G. 1998. Hysteresis in magnetism. San Diego: Academic Press.

Bonevich JE, Harada K, Matsuda T, Kasai H, Yoshida T, Pozzi G, Tonomura A. 1993. Electron holography observation of vortex lattices in a superconductor. Phys Rev Lett 70:2952-2955.

Champion Y, Bigot J. 1996. Preparation and characterization of nanocrystalline copper powders. Scripta Mat 35:517-522.

Champion Y, Langlois C, Guerin-Mailly S, Langlois P, Bonnentien JL, Hytch MJ. 2003. Near-perfect elastoplasticity in pure nanocrystalline copper. Science 300:310-311.

Connerney JEP, Acuña MH, Wasilewski PJ, Ness NF, Reme H, Mazelle C, Vignes, D, Lin RP, Mitchell DL, Cloutier PA. 1999. Magnetic lineations in the ancient crust of Mars. Science 284:794-798. Cowburn RP, Koltsov DK, Adeyeye AO, Welland ME, Tricker DM. 1999. Single-domain circular nanomagnets. Phys Rev Lett 83:10421045.

de Graef M, Nuhfer NT, McCartney MR. 1999. Phase contrast of spherical magnetic particles. J Microsc 194:84-94.

Dunin-Borkowski RE, McCartney MR, Smith DJ, Parkin SSP. 1998. Towards quantitative electron holography of magnetic thin films using in situ magnetization reversal. Ultramicroscopy 74:61-73.

Dunin-Borkowski RE, McCartney MR, Kardynal B, Parkin SSP, Scheinfein MR, Smith DJ. 2000. Off-axis electron holography of patterned magnetic nanostructures. J Microsc 200:187-204.

Dunin-Borkowski RE, McCartney MR, Posfai M, Frankel RB, Bazylinski DA, Buseck PR. 2001. Off-axis electron holography of magnetotactic bacteria: magnetic microstructure of strains MV-1 and MS-1. Eur J Mineral 13:671-684.

Dunin-Borkowski RE, McCartney MR, Smith DJ. 2004. Electron holography of nanostructured materials. In: Nalwa HS, editor. Encyclopaedia of nanoscience and nanotechnology, vol. 3. Stevenson Ranch, CA: American Scientific Publishers. p 41-100.

Dunlop DJ, Özdemir Ö. 1997. Rock magnetism, fundamentals and frontiers. Cambridge: Cambridge University Press.

Ghiglia DC, Pritt MD. 1998. Two-dimensional phase unwrapping theory, algorithms and software. New York: John Wiley \& Sons.

Harrison RJ, Dunin-Borkowski RE, Putnis A. 2002. Direct imaging of nanoscale magnetic interactions in minerals. Proc Natl Acad Sci USA 99:16556-16561.

Harscher A, Lichte H. 1996. Experimental study of amplitude and phase detection limits in electron holography. Ultramicroscopy 64 : $57-66$.

Hytch MJ, Dunin-Borkowski RE, Scheinfein MR, Moulin J, Duhamel C, Mazelayrat F, Champion Y. 2003. Vortex flux channeling in magnetic nanoparticle chains. Phys Rev Lett 91:257207.

Jacobs IS, Bean CP. 1955. An approach to elongated fine-particle magnets. Phys Rev 100:1060-1067.

Lai GM, Hirayama T, Fukuhara A, Ishizuka K, Tanji T, Tonomura A. 1994. 3-dimensional reconstruction of magnetic vector-fields using electron-holographic interferometry. J Appl Phys 75:4593-4598.

Lichte H. 1995. State and experimental steps towards $0.1 \mathrm{~nm}$ with the CM30-Special Tübingen. In: Tonomura A, Allard LF, Pozzi G, Joy DC, Ono YA, editors. Electron holography. Amsterdam: Elsevier. p 11-31.

Lichte H, Herrmann KH, Lenz F. 1987. Electron noise in off-axis image plane holography. Optik 77:135-140.

Loudon JC, Mathur ND, Midgley PA. 2002. Charge-ordered ferromagnetic phase in $\mathrm{La}_{0.5} \mathrm{Ca}_{0.5} \mathrm{MnO}_{3}$. Nature 420:797-800.

Matteucci G, Muccini M, Hartmann U. 1994. Flux measurements on ferromagnetic microprobes by electron holography. Phys Rev B $50: 6823-6828 \mathrm{~d}$.

McCartney MR, Zhu Y. 1998. Induction mapping of $\mathrm{Nd}_{2} \mathrm{Fe}_{14} \mathrm{~B}$ magnetic domains by electron holography. Appl Phys Lett 72:1380-1382.

Midgley PA, Weyland M, Thomas JM, Johnson BFG. 2001. Z-contrast tomography: a technique in three-dimensional nanostructural analysis based on Rutherford scattering. Chem Commun 10:907-908.

O'Handley RC. 2000. Modern magnetic materials. New York: Wiley.

Osakabe N, Yoshida K, Horiuchi Y, Matsuda T, Tanabe H, Okuwaki T, Endo J, Fujiwara H, Tonomura A. 1983. Observation of recorded magnetization pattern by electron holography. Appl Phys Lett 42: $746-748$.

Price GD. 1981. Subsolidus phase-relations in the titanomagnetite solid-solution series. Am Mineral 66:751-758.

Seraphin S, Beeli C, Bonard JM, Jiao J, Stadelmann PA, Chatelain A. 1999. Magnetization of carbon-coated ferromagnetic nanoclusters determined by electron holography. J Mater Res 14:2861-2870. 
Signoretti S, Del Blanco L, Pasquini L, Matteucci G, Beeli C, Bonetti E. 2003. Electron holography of gas-phase condensed Fe nanoparticles. J Magn Magn Mat 262:142-145.

Snoeck E, Dunin-Borkowski RE, Dumestre F, Renaud P, Amiens C, Chaudret B, Zurcher P. 2003. Quantitative magnetization measurements on nanometer ferromagnetic cobalt wires using electron holography. Appl Phys Lett 82:88-90.

Sugawara A, Streblechenko D, McCartney MR, Scheinfein MR. 1998. Magnetic coupling in self-organized narrow-spaced $\mathrm{Fe}$ nanowire arrays. IEEE Trans Magn 34:1081-1083.

Tonomura A. 1992. Electron-holographic interference microscopy. Adv Phys 41:59-103.

Tonomura A, Matsuda T, Endo J, Arii T, Mihama K. 1980. Direct observation of fine structure of magnetic domain walls by electron holography. Phys Rev Lett 44:1430-1433.
Tonomura A, Osakabe N, Matsuda T, Kawasaki T, Endo J, Yano S, Yamada H. 1986. Evidence for Aharonov-Bohm effect with magnetic field completely shielded from electron wave. Phys Rev Lett 56:792-795.

Tripp SL, Pusztay SV, Ribbe AE, Wei A. 2002. Self-assembly of cobalt nanoparticle rings. J Am Chem Soc 124:7914-7915.

Tripp SL, Dunin-Borkowski RE, Wei A. 2003. Flux closure in selfassembled nanoparticle rings. Angew Chem 42:5591-5593.

Wachowiak A, Wiebe J, Bode M, Pietzsch O, Morgenstern M, Wiesendanger R. 2002. Direct observation of internal spin-structure of magnetic vortex cores. Science 298:577-580.

Williams W, Wright TM. 1998. High-resolution micromagnetic models of fine grains of magnetite. J Geophys Res Solid Earth 103:30537-30550.

Zhu J-G, Zheng Y, Prinz GA. 2000. Ultrahigh density vertical magnetoresistive random access memory. J Appl Phys 87:6668-6673. 